\title{
Stochastic Hopf bifurcations in vacuum optical tweezers
}

\author{
Stephen H. Simpson $\odot,{ }^{1, *}$ Yoshihiko Arita, ${ }^{2,3}$ Kishan Dholakia, ${ }^{2,3,4,5}$ and Pavel Zemánek ${ }^{1}$ \\ ${ }^{1}$ Institute of Scientific Instruments of the Czech Academy of Sciences, v.v.i., Královopolská 147, 61264 Brno, Czech Republic \\ ${ }^{2}$ SUPA, School of Physics and Astronomy, University of St Andrews, North Haugh, St Andrews KY16 9SS, United Kingdom \\ ${ }^{3}$ Molecular Chirality Research Centre, Chiba University, 1-33 Yayoi-cho, Inage-ku, Chiba-shi 263-0022, Japan \\ ${ }^{4}$ College of Optical Sciences, The University of Arizona, Tucson, Arizona 85721-0094, USA \\ ${ }^{5}$ Department of Physics, College of Science, Yonsei University, Seoul 03722, South Korea
}

(Received 8 June 2021; accepted 23 September 2021; published 15 October 2021)

\begin{abstract}
The forces acting on an isotropic microsphere in optical tweezers are effectively conservative. However, reductions in the symmetry of the particle or trapping field can break this condition. Here we theoretically analyze the motion of a particle in a linearly nonconservative optical vacuum trap, concentrating on the case where symmetry is broken by optical birefringence, causing nonconservative coupling between rotational and translational degrees of freedom. Neglecting thermal fluctuations, we first show that the underlying deterministic motion can exhibit a Hopf bifurcation in which the trapping point destabilizes and limit cycles emerge whose amplitude grows with decreasing viscosity. When fluctuations are included, the bifurcation of the underlying deterministic system is expressed as a transition in the statistical description of the motion. For high viscosities, the probability distribution is normal, with a kurtosis of three, and persistent probability currents swirl around the stable trapping point. As the bifurcation is approached, the distribution and currents spread out in phase space. Following the bifurcation, the probability distribution function hollows out, reflecting the underlying limit cycle, and the kurtosis halves abruptly. The system is seen to be a noisy self-sustained oscillator featuring a highly uneven limit cycle. A variety of applications, from autonomous stochastic resonance to synchronization, is discussed.
\end{abstract}

DOI: 10.1103/PhysRevA.104.043518

\section{INTRODUCTION}

An object held in optical tweezers is usually thought to be resting in a potential well [1,2], formed by the concentrated light intensity found in a tightly focused optical beam. Under certain circumstances, described further below, this is a good approximation which leads to a number of useful applications. For example, the trap is at thermodynamic equilibrium and the particle coordinates are distributed according to Boltzmann statistics, e.g., $P(x) \propto \exp \left[-V(x) / k_{B} T\right]$, for potential $V(x)$. This ensures that the particle is confined by the potential and that deepening the well by, for instance, increasing the laser power results in the particle being more tightly confined. Providing the particle remains in the linear range of the trap, we can apply the equipartition of energy to associate the elastic energy of the trap, $K\left\langle x^{2}\right\rangle / 2$ (where $K$ is a scalar stiffness and $\left\langle x^{2}\right\rangle$ is the variance in the position), with the thermal energy, $k_{B} T / 2$. Thus the variance in the position is inversely proportional to the stiffness which, in turn, is proportional to the laser power, i.e., $\left\langle x^{2}\right\rangle=k_{B} T / P k$ (where $P$ is the power and $k=K / P$ is the stiffness per unit power) $[3,4]$. Notably, this analysis applies in both under- and overdamped regimes, i.e., time-averaged quantities, such as $\left\langle x^{2}\right\rangle$, are independent of the viscosity of the ambient medium.

\footnotetext{
*simpson@isibrno.cz
}

The description given above assumes that the optical forces provided by the tweezer are conservative, i.e., that they are given by the gradient of a scalar potential. Countless studies confirm the validity of this assumption when applied to spherical particles making small excursions in linearly polarized Gaussian traps, e.g., Refs. [5,6]. More recent work shows that the optical forces are locally conservative in this particular system, for reasons of symmetry rather than any more fundamental requirement [7-12] and, furthermore, that the nonconservative character of optical forces manifests itself whenever the symmetry of the system is lowered or when the particle roams widely in a weak trap $[13,14]$. In the overdamped regime, this results in persistent probability currents connected with a subtle, systematic bias in the Brownian motion of the trapped object [15]. In the underdamped regime, where inertia is significant, the consequences are more dramatic. This systematic bias increases the particle momentum. Reducing the ambient viscosity reinforces this effect, expanding the amplitude of the stochastic motion. Eventually, the trapping point destabilizes, as the nonconservative forces exponentially amplify any small perturbation. Similar effects have been observed for isotropic microspheres in circularly polarized beams [16] and for birefringent spheres in linearly polarized beams [17]. In the former case, symmetry is broken by inhomogeneous optical spin, which generates transverse momentum circulating about the beam axis. In the latter, optical anisotropy introduces nonsymmetric coupling between rotational and translational degrees of freedom: tilting the 
particle in the beam generates transverse forces (similarly to other systems $[11,18,19])$, while displacing the particle generates a torque as well as a restoring force. These processes are nonsymmetric, so that a closed path of alternate rotations and translations involves an exchange of energy with the environment. To some degree, these physical processes operate in all lower symmetry vacuum traps, including those containing nonspherical particles, e.g., Refs. [20-23]. The current paper expands on previous work describing the motion of birefringent spheres in linearly polarized traps [17], which focused on stochastic motion in the linear regime as the instability is approached.

Here, we theoretically consider the general motion, before and after the instability, including the behavior that depends explicitly on nonlinearities in the force field. In order to draw out the effect of thermal fluctuations, we separately consider the deterministic case, which includes viscous damping but ignores thermal fluctuations, and the equivalent stochastic system, where fluctuations are properly accounted for. These two cases are referred to as the equivalent stochastic and deterministic systems.

To start with, we concentrate on the deterministic system. We show that the nonconservative instability described in [17] corresponds to a Hopf bifurcation wherein the stable trapping point is replaced by limit cycles whose amplitude increases as the ambient viscosity $\mu$ is decreased beneath a critical value $\mu_{X}$. We identify the limit cycles numerically and characterize them in terms of energy changes around the cycle.

In the second part of the paper, we include thermal fluctuations. Prior to the bifurcation (viscosity greater than the critical value, $\mu>\mu_{X}$ ), the particle remains within the linear range of the trap. As a consequence, the probability distribution function is normal [24] and can be evaluated analytically, along with the probability currents which appear due to the nonconservative part of the force. For sufficiently high viscosities, the probability distribution resembles the Boltzmann distribution. The variance of the coordinates is equivalent to the values that would be obtained if the nonconservative part of the force field was neglected, and the variance in the velocity is approximately given by the thermal velocity, e.g., $\left\langle v^{2}\right\rangle \approx k_{B} T / m$, for mass $m$. However, the distribution contains covariances which would vanish identically if the system really was conservative. In particular, some of the position coordinates are correlated with some of the velocities. These couplings are connected with probability currents that swirl around the trapping point. As the viscosity is reduced and the bifurcation is approached, the distribution function spreads out in phase space, but remains normal. The probability currents decrease in amplitude and spread out. After the bifurcation, the spreading distribution flattens and, eventually, hollows out, characterizing a noisy, self-sustained oscillation. The bifurcation itself is associated with an abrupt change in the kurtosis of the probability distribution function, from a value of 3 (i.e., normal distribution) to a value of $\approx 1.5$.

Finally, we briefly discuss a broader range of physical systems (including neural $[25,26]$ and laser dynamics $[27,28]$ ) that share stochastic and dynamical features with the optomechanical system featured here, as well as the stochastic phenomena that these systems can exhibit.

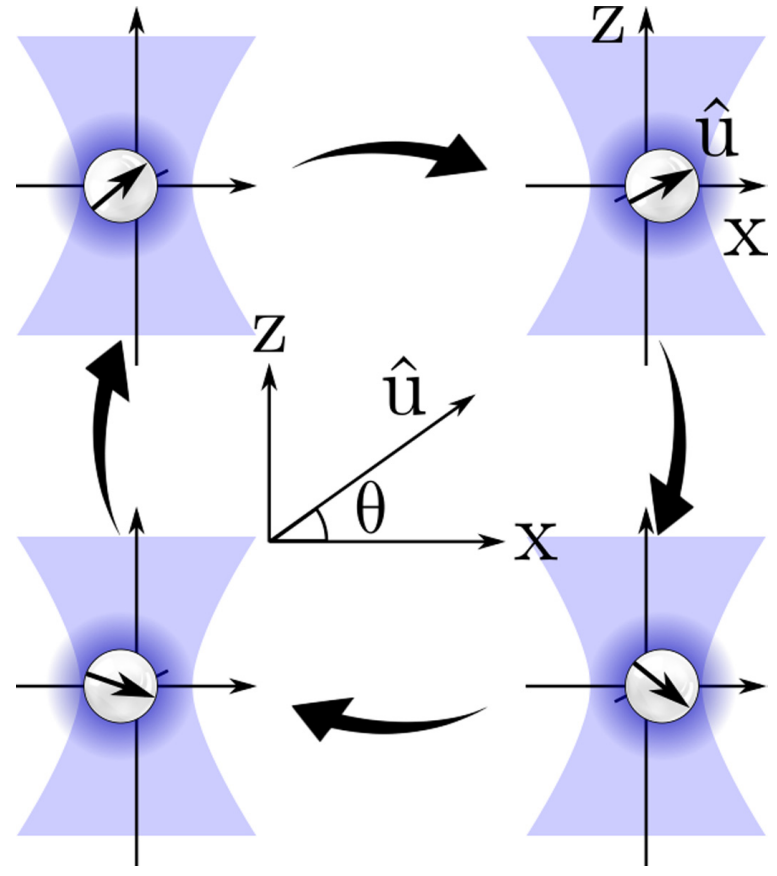

FIG. 1. Schematic showing the coordinate scheme used throughout the paper and a closed cycle of repeated rotations and translations.

In the following sections, we review background material, and describe the relevant equations of motion and the form of the force field before analyzing the deterministic and stochastic motion.

\section{BACKGROUND}

We consider a system identical to that described previously [17]. The coordinates and geometry are shown in Fig. 1. A positively birefringent microsphere is levitated in a linearly polarized optical trap in a vacuum chamber. The microsphere has a unique symmetry axis, $u$ in Fig. 1, corresponding to the optic axis. The trapping beam is directed upwards so that the radiation pressure is balanced by the weight of the particle. At the trapping point, where the external forces on the particle vanish, the symmetry axis of the particle aligns with the polarization direction. The angle between $u$ and the $x y$ plane is measured by $\theta$. We are interested in how the motion of the microsphere changes as the viscosity is reduced. In an experiment, this is achieved by lowering the pressure in the vacuum chamber. We avoid discussion of the relationship between pressure and viscosity and simply quote viscosity since this is the quantity appearing in the equations of motion. Experimental measurements of viscosity for varying pressure have been given previously $[17,29]$ provides a commonly used theoretical approximation. As suggested above, the bifurcation parameter is the viscosity $\mu$ and the system bifurcates when $\mu=\mu_{X}$, the critical value. Viscosities are therefore given as ratios with $\mu_{X}$.

Unless stated otherwise, the following physical parameters are used in all calculations and simulations. The particle is a birefringent sphere with radius $a=2.2 \mu \mathrm{m}$, density $\rho=$ $2.54 \mathrm{~g} / \mathrm{cm}^{3}$, and refractive indices $n_{o}=1.65$ and $n_{e}=1.55$, 
according to the bulk parameters for vaterite. The trapping Gaussian beam is linearly polarized in the $x$ direction with wavelength $1064 \mathrm{~nm}$, focused with a numerical aperture of 0.95 , with power $5 \mathrm{~mW}$. As shown below, the critical viscosity for these parameters is $\mu_{X}=1.98 \times 10^{-6} \mathrm{~Pa} \mathrm{~s}$, which corresponds approximately to a chamber pressure of 1 mbar. Next we discuss the equations of motion and the nature of the force field acting on the particle.

\section{A. Langevin equation}

The motion of the particle is determined by the following Langevin equation:

$$
\mathbf{f}^{\mathrm{opt}}(\mathbf{r})+\mathbf{f}^{L}(t)-m g \hat{\mathbf{z}}-\mathbf{\Xi} \dot{\mathbf{r}}=\mathbf{M} \ddot{\mathbf{r}} .
$$

Here, $\mathbf{f}^{\text {opt }}(\mathbf{r})$ is the generalized optical force (i.e., forces and torques) acting on the particle at position $\mathbf{r}$, where $\mathbf{r}$ specifies the coordinates of the center of mass and the orientation. In this article, we are concerned with a nonlinear force field which is nonconservative at first order and above; see below. $\Xi$ is the pressure-dependent friction matrix with diagonal elements $\Xi_{x x} \equiv \xi_{x}=6 \pi \mu a$ and $\Xi_{\theta \theta} \equiv \xi_{\theta}=8 \pi \mu a^{3}$ for translational and rotational motion, respectively. $\mathbf{f}^{L}$ is the stochastic, Langevin force, uncorrelated, with zero mean and amplitude fixed by the fluctuation-dissipation theorem, i.e., $\left\langle f_{i}^{L}(t)\right\rangle=0,\left\langle f_{i}^{L}(t) f_{j}^{L}\left(t^{\prime}\right)\right\rangle=2 k_{B} T \Xi_{i j} \delta\left(t-t^{\prime}\right)$, for orthogonal components $i, j$. M is a diagonal matrix whose elements are given by the mass $(m)$ of the sphere and its moment of inertia $(I)$, and $-m g \hat{\mathbf{z}}$ is the weight.

We treat the motion of the particle in several different ways. For viscosities above the critical value, $\mu>\mu_{X}$, the trapping point is stable and the forces encountered by the particle are approximately linear. Under these conditions, deterministic trajectories ( $\mathbf{f}^{L}$ set to zero) can be found analytically. In addition, probability distributions and currents for the associated thermal motion ( $\mathbf{f}^{L}$ is the usual Langevin force) can also be computed directly. Below threshold $\left(\mu<\mu_{X}\right)$, the particle motion exceeds the linear range of the trap and the motion is simulated using an established numerical scheme [30], generalized to include rotation [17]. This is sufficient to explore the stochastic motion. To analyze the underlying deterministic motion, we simply set the Langevin force $\mathbf{f}^{L}$ to zero and use the same numerical scheme. This procedure provides initial approximations to the limit cycles formed below threshold, which are subsequently refined using a variational approach [31]. Further comments about all of the methods are provided at appropriate junctures below.

\section{B. Forces and torques}

For the system under consideration, the phenomena of interest are generated by nonsymmetric coupling between translational and rotational degrees of freedom [17]. This coupling is intrinsically nonconservative so that a closed loop consisting of repeated rotations and translations will generally involve an exchange of energy between the optical field and the particle. This particular geometry allows us to restrict attention to two coordinates, $x$ and $\theta$ (see Fig. 1). There are several reasons for this. First, the symmetry of the system requires that all other optical forces and torques are uncoupled at first order [17]. Second, rotational degrees of freedom are uncoupled in the body frame since the moment of inertia is isotropic. Third, the symmetry axis of the particle, $\hat{\mathbf{u}}$, is tightly bound in the $x z$ plane by the interaction with the polarization. Optical torques about $\hat{\mathbf{u}}$ vanish identically [32] so that the particle diffuses freely about this axis. As a result, the angular momentum about $\hat{\mathbf{u}}$ is too small and too irregular to cause precession [21] which, in addition, is suppressed by the confinement of $\hat{\mathbf{u}}$ to the $x z$ plane. To good approximation, each of the uncoupled degrees of freedom acts as a separate one-dimensional subsystem which is effectively conservative (due to its dimension) and satisfies equipartition. The one exception to this is the $z$ coordinate, which couples with $x$ at second order. This effect is relatively minor and is discussed in the conclusions.

To quantify our restricted force field, we perform generalized Lorentz-Mie calculations for a birefringent sphere with the given parameters $[17,33]$. Optical forces $f_{x}$ and torques $t_{y}$ are computed on a fine lattice of points in the range $-1 \leqslant x \leqslant$ $1 \mu \mathrm{m}$ and $-\pi / 4 \leqslant \pi / 4$; see Fig. 2 . The strongest nonlinearity is in the variation of $f_{x}$ with $\theta$. Otherwise, these forces are quite close to linear.

To aid analysis and simulation, we fit the exact forces and torques (Fig. 2) with odd, low-order polynomials,

$$
\begin{aligned}
& f_{x}(x, \theta) \approx-k_{x}^{(x)} x-k_{\theta}^{(x)} \theta-\sum_{n=0,3} \chi_{n}^{(x)} x^{3-n} \theta^{n}, \\
& t_{y}(x, \theta) \approx-k_{x}^{(\theta)} x-k_{\theta}^{(\theta)} \theta-\sum_{n=0,3} \chi_{n}^{(\theta)} x^{3-n} \theta^{n} .
\end{aligned}
$$

These approximations fit the data quite accurately with maximum errors of less than $5 \%$. Numerical values for the various coefficients are given in the Appendix. We note that the simulations described below can also be performed using forces and torques evaluated by interpolating the data depicted in Fig. 2. There are minor discrepancies between the results obtained using Eqs. (2a) and (2b) and those obtained with interpolated forces. These differences are discussed below. They are negligible in comparison with experimental uncertainties and leave the results qualitatively unaltered.

\section{DETERMINISTIC MOTION}

In this section, we consider the deterministic motion of the birefringent sphere in the absence of thermal fluctuations. First we analyze the stability of the trapping point (the point at which the external forces vanish) for varying viscosity, and state the conditions under which stability is lost. Next, we consider the motion for viscosities below the critical value, $\mu<\mu_{X}$, where the stable fixed point becomes a repelling fixed point. For purely linear force fields, all trajectories spiral outwards and the particle is expelled from the trap. In reality, small curvatures result in the formation of stable limit cycles whose amplitude increases with decreasing viscosity. These limit cycles are identified by integrating the equation of motion, given by Eq. (1), with forces, given by Eq. (2), and confirmed using a variational principle. 

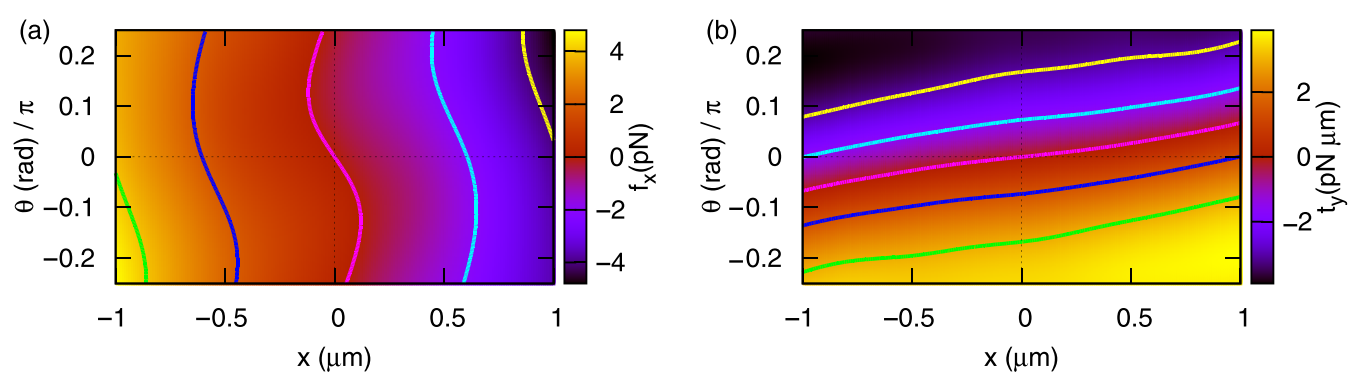

FIG. 2. (a) The $x$ component of the optical force, $f_{x}(x, \theta)$, and (b) the $y$ component of the torque, $t_{y}(x, \theta)$. In (a), the contours correspond to $f_{x}=4,2,0,-2,-4 \mathrm{pN}$ from left to right, and in (b), the contours are at $t_{y}=-3,-1.5,0,1.5,3 \mathrm{pN} \mu \mathrm{m}$ from top to bottom.

\section{A. Linear stability}

An analysis of the linear stability of this system has been presented previously [17]. A summary is included here for completeness. Omitting the stochastic force and linearizing the equation of motion (1), with forces (2), gives

$$
-\mathbf{k x}-\boldsymbol{\Xi} \dot{\mathbf{x}}=\mathbf{M} \ddot{\mathbf{x}} \Longleftrightarrow\left(\mathbf{k}+i \omega \boldsymbol{\Xi}-\mathbf{M} \omega^{2}\right) \mathbf{X} \equiv \mathbf{A X}=0,
$$

where $\mathbf{k}$ is the matrix of the linear stiffness coefficients, $k_{x / \theta}^{(x / \theta)}$, in Eq. (2) and $\mathbf{x}=(x, \theta)$ are the spatial coordinates of the restricted system.

The right-hand side term of Eq. (3) is the Fourier transform of the left-hand side and pertains to solutions of the form $\mathbf{x} \sim \mathbf{X} e^{i \omega_{i} t}$ with $\mathbf{X}=(X, \Theta)$. Setting the determinant $|\mathbf{A}|=0$ yields the four characteristic frequencies, $\omega_{i}, i=1, \ldots, 4$. Since the friction $\Xi \propto \mu$, the characteristic frequencies also vary with viscosity $\mu$. In this regime, trajectories are superpositions of terms, $\sim \mathbf{X}_{0, i} e^{i \operatorname{Re}\left(\omega_{i}\right) t} e^{-\operatorname{Im}\left(\omega_{i}\right) t}$, for each $\omega_{i}$ and the vector amplitudes $\mathbf{X}_{0, i}$ are determined by the initial conditions. If the imaginary parts of each of the characteristic frequencies are positive [i.e., $\operatorname{Im}\left(\omega_{i}\right)>0 \forall i$ ], the trajectories spiral into the origin. If one or more of the $\operatorname{Im}\left(\omega_{i}\right)$ are negative, trajectories spiral outwards, in general, and the trap is unstable within the linear approximation.

A condition for the existence of this instability can be derived by considering the behavior at $\mu=0$. If the trap is unstable for $\mu=0$, then it must lose stability for some critical viscosity, $\mu=\mu_{X}$. Examination of the values of $\omega_{i}$ at $\mu=0$ yields the following condition for the trap to destabilize for some positive value of $\mu_{X}$ :

$$
\Delta_{L P}=\frac{1}{4}\left(\frac{k_{\theta}^{(\theta)}}{I}-\frac{k_{x}^{(x)}}{m}\right)^{2}+\frac{k_{\theta}^{(x)} k_{x}^{(\theta)}}{m I}<0,
$$

where $\mu_{X}$ itself is given by

$s_{X} s_{\theta} \mu_{X}^{2}=m I \omega_{X}^{2}-\left(m k_{\theta}^{(\theta)}+I k_{x}^{(x)}\right)+\left(k_{x}^{(x)} k_{\theta}^{(\theta)}-k_{\theta}^{(x)} k_{x}^{(\theta)}\right) / \omega_{X}^{2}$, where the friction coefficients $\xi_{x}=6 \pi \mu a \equiv s_{x} \mu, \quad \xi_{\theta}=$ $8 \pi \mu a^{3} \equiv s_{\theta} \mu$ are proportional to the viscosity, and $\omega_{X}$ is the corresponding real part of the frequency,

$$
\omega_{X}^{2}=\frac{k_{x}^{(x)} s_{\theta}+k_{\theta}^{(\theta)} s_{x}}{m s_{\theta}+I s_{x}} .
$$

The stiffness coefficients [see Eq. (2) and the Appendix] satisfy the condition given by Eq. (4), and the resulting frequencies $\omega_{i}$ have the form shown in Fig. 3. For the default parameters, defined above, we have $\mu_{X}=1.98 \times 10^{-6} \mathrm{~Pa} \mathrm{~s}$. Two further frequencies exist. These have the same imaginary parts as those shown in the figure, while the real parts have the opposite sign to the values in the graph. As can be seen, the real frequencies are relatively constant and one of the imaginary values changes sign at $\mu_{X}$.

\section{B. Hopf bifurcation and limit cycles}

Any solution of the linear system, given by Eq. (3), is a linear combination of terms, $\sim \mathbf{X}_{i} e^{i \omega_{i} t}$, where $\mathbf{X}_{i}$ is a vector amplitude. As soon as $\mu<\mu_{X}$, all trajectories are dominated by the exponentially growing term and no trajectories are confined. In reality, the optical beam is of finite extent and the nonlinear terms in the force field, given by Eq. (2), have a qualitative influence on the motion. Instead of spiraling ever outward, periodic motion, described by a limit cycle, emerges, effectively confining the particle. This is an unusual scenario for optical tweezers. Usually, we expect that a tweezed particle is trapped by linear terms in the force field. In this case, the linearized force is unstable and the particle is confined by nonlinearities.

We investigate this behavior by numerically integrating the equations of motion [17,30], with approximate force field (2), setting the Langevin force to zero. After the simulation has

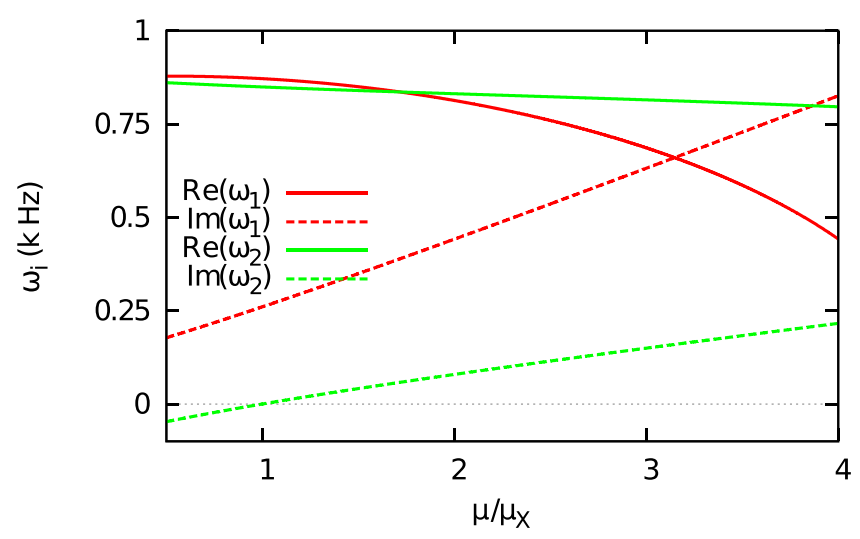

FIG. 3. Eigenfrequencies $\omega_{1,2}$ as functions of $\mu / \mu_{X} \cdot \operatorname{Im}\left(\omega_{2}\right)$ changes sign at $\mu=\mu_{X}$, destabilizing the trapping position. 

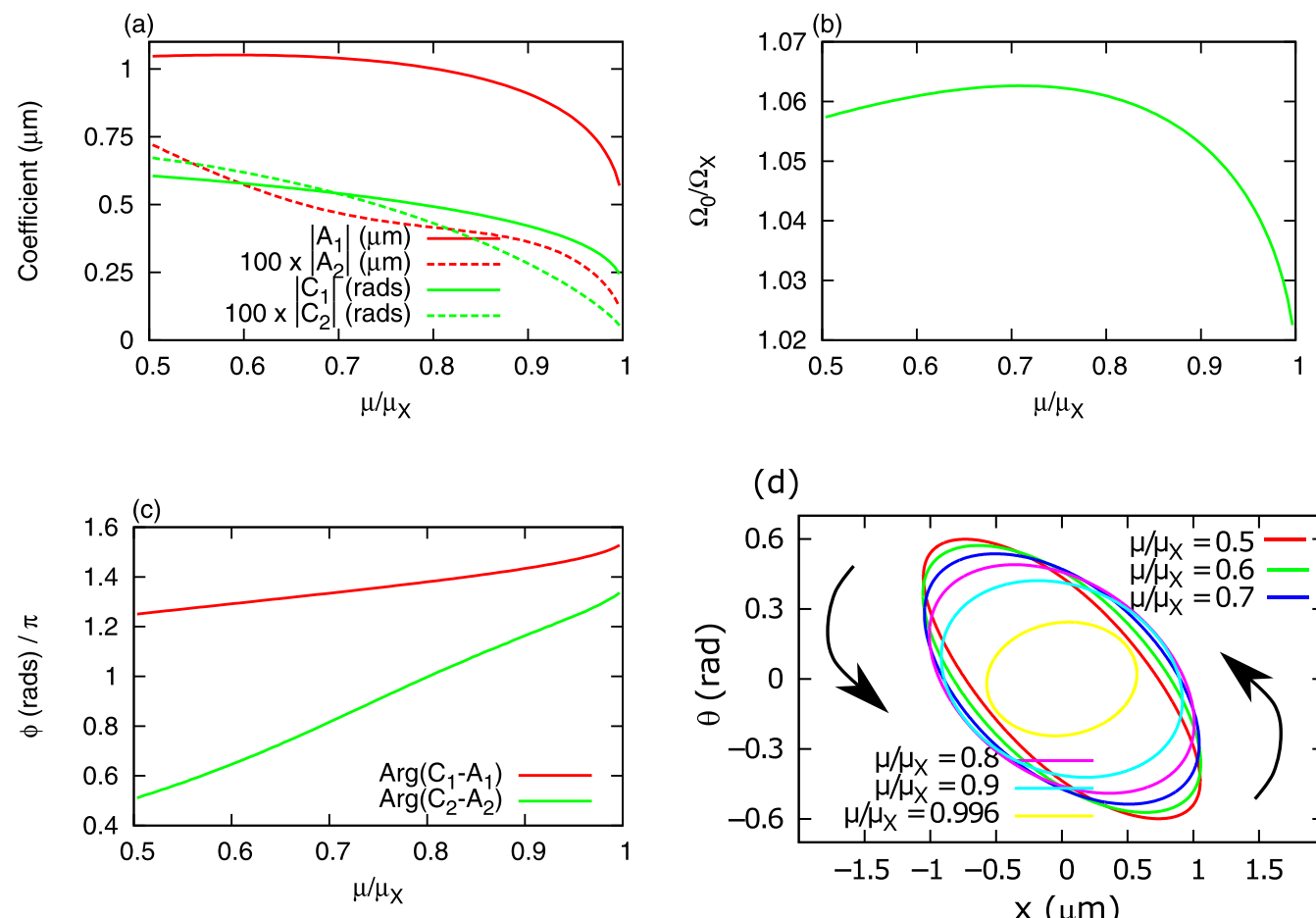

(d)

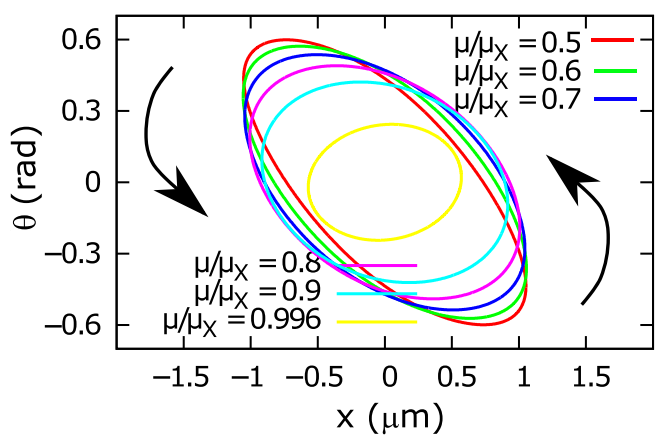

FIG. 4. (a) Amplitudes of the Fourier coefficients of the limit cycle expansions, strongly dominated by the fundamental oscillations with amplitudes $\left|A_{1}\right|$ and $\left|C_{1}\right|$. (b) Variation of the fundamental frequency $\left(\boldsymbol{\Omega}_{0}\right)$ relative to the threshold value $\left(\boldsymbol{\Omega}_{X}\right)$, with $\mu / \mu_{X}$. (c) Relative phases of rotational and translational oscillation for the fundamental and third harmonic. (d) $(x, \theta)$ coordinates for limit cycles with various $\mu / \mu_{X}$. The arrows indicate the sense in which the cycle is traversed. The smallest, inner cycle corresponds to $\mu / \mu_{X}=0.996$ and the greatest has $\mu / \mu_{X}=0.5$.

reached the steady state, we fit the trajectory with a Fourier series with fundamental frequency, $\boldsymbol{\Omega}_{0}$,

$$
\begin{aligned}
& x(t)=\operatorname{Re}\left\{\sum_{n=1, N} A_{n} \exp \left[-i(2 n-1) \boldsymbol{\Omega}_{0} t\right]\right\}, \\
& \theta(t)=\operatorname{Re}\left\{\sum_{n=1, N} C_{n} \exp \left[-i(2 n-1) \boldsymbol{\Omega}_{0} t\right]\right\} .
\end{aligned}
$$

A variational approach is used to improve accuracy and confirm that the trajectory is indeed a limit cycle. Following [31], we numerically find solutions for the equations,

$$
\int_{0}^{2 \pi / \mathbf{\Omega}_{0}} \sum_{i=x, \theta} \mathbf{P}_{i} \cdot \frac{\partial q_{i}}{\partial \alpha_{j}} d t=0,
$$

where $P_{i}$ are the generalized forces which, in this case, reduce to $f_{x}-m \ddot{x}$ for translations and $t_{y}-I \ddot{\theta}$ for rotations, $q_{i}$ are the generalized coordinates [which reduce to $x\left(\left\{\alpha_{i}\right\}\right)$ and $\left.\theta\left(\left\{\alpha_{i}\right\}\right)\right]$, $\alpha_{i}=\left\{A_{i}, C_{i}, \boldsymbol{\Omega}_{0}\right\}$ are the parameters defining the periodic oscillation, and $N$ indicates the highest-order term in the expansion. Equations (7) form a set of nonlinear equations, one for each of the $N$ parameters, $\left\{\alpha_{j}\right\}$. The derivatives are easily evaluated with standard computer algebra [34], allowing the solution to be sought with multivariate Newton-Raphson. We note that this approach is quite cumbersome. The algebraic equations, while simple, are long and unwieldy for the moderate values of $N$ used here (we use $N=4$, which is more than sufficient). We note that the variational approach does not significantly improve the accuracy obtained by fitting Eqs. (6) and (6b) and, in this respect, it is unnecessary. However, it does provide reliable confirmation that we have found true limit cycles, not weakly quasiperiodic oscillations resembling limit cycles, and we include discussion of this method for that reason.

As the ambient viscosity is reduced, the limit cycles tend to increase in amplitude. In terms of the Fourier parameters, this variation is shown in Fig. 4. The first two Fourier coefficients, $A_{1}, C_{1}, A_{2}, C_{2}$, are shown in Fig. 4(a). Although the nonlinearity of the force field is necessary for the confinement of the particle, the periodic oscillation is dominated by the fundamental, the amplitude of which is about two orders of magnitude greater than the next highest harmonic. In Fig. 4(b), we see that the fundamental frequency, which takes a value $\boldsymbol{\Omega}_{X}$ at the bifurcation point, varies only slightly with viscosity. Figure 4(c) shows the relative phase between the $x$ and $\theta$ oscillations for the fundamental and third harmonic. For $\mu$ just less than $\mu_{X}$, the phase difference for the fundamental oscillation is $\sim \pi / 2$, so that the limit cycle is approximately circular in phase space. As $\mu$ decreases, the relative phase drops and the cycle becomes increasingly anisotropic. Finally, Fig. 4(d) shows the limit cycle itself in $(x, \theta)$ space for a range of values of $\mu / \mu_{X}$ : the limit cycle grows in amplitude, becoming increasingly anisotropic and tilted as $\mu$ is decreased beneath $\mu_{X}$.

Over one complete cycle, the energy dissipated through viscous damping [Eq. (8a)] must be exactly compensated by energy transferred to the particle from the 

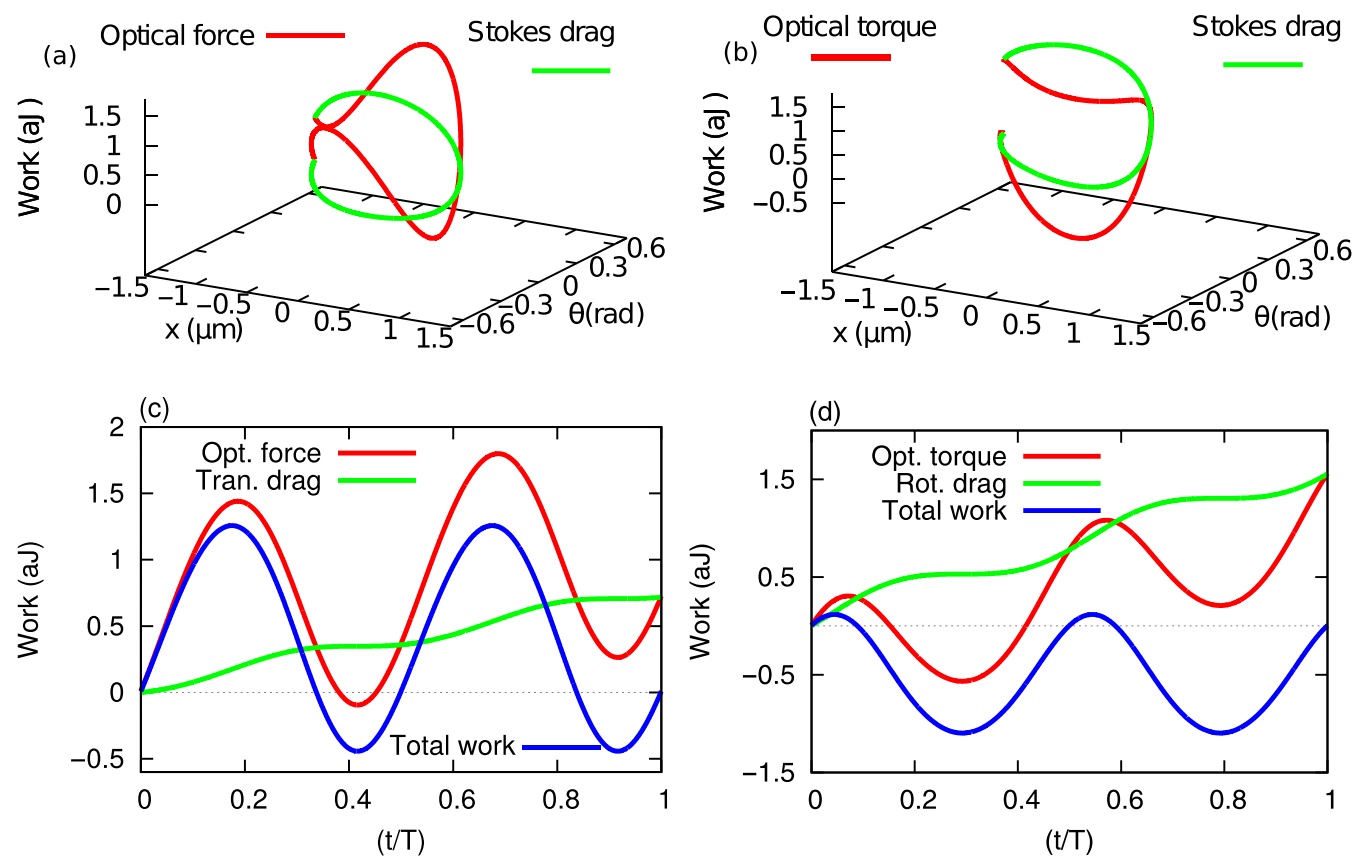

FIG. 5. (a), (c) Translational and (b), (d) rotational work done around a sample limit cycle with $\mu / \mu_{X}=0.5$. The work done against the optical field is separated from the work done by viscous drag. (a), (b) The work done as a function of $x$ and $\theta$. (c), (d) The variation with time $(t)$ over one complete cycle with period $(T)$. As the particle traverses the limit cycle, it dissipates energy into the environment through viscous drag. On completion of the cycle, the dissipated energy is exactly compensated for by energy added to the system by optical forces so that the total work done over the cycle is zero.

optical field [Eq. (8b)], so that the total change in energy is zero,

$$
\begin{aligned}
W_{x}^{(\mathrm{visc})}(t) & =\int_{0}^{t} d t \xi_{x}|\boldsymbol{v}|^{2}, W_{\theta}^{(\mathrm{visc})}(t)=\int_{0}^{t} d t \xi_{\theta}|\boldsymbol{\Omega}|^{2}, \\
W_{x}^{(\mathrm{opt})}(t) & =\int_{0}^{t} d t \mathbf{f} \cdot \mathbf{v}, W_{\theta}^{(\mathrm{opt})}(t)=\int_{0}^{t} d t \boldsymbol{\tau} \cdot \boldsymbol{\Omega},
\end{aligned}
$$

where $\boldsymbol{v}$ is the velocity, $\boldsymbol{\Omega}$ is the angular velocity, given by the time derivatives of the position and orientation, and $f$ and $\boldsymbol{\tau}$ are the force and torque vectors. $W_{x / \theta}^{(v i s c) /(o p t)}$ is the work done by the viscous or optical forces for translational $(x)$ or rotational $(\theta)$ motion.

This balance must be separately satisfied by translational and rotational motion. This is demonstrated in Fig. 5, which shows the integrated energy due to optical forces (red lines) and viscous forces (green lines) for translational [Figs. 5(a) and 5(c)] and rotational [Figs. 5(b) and 5(d)] work when the relative viscosity is $\mu / \mu_{X}=0.5$. Figures 5(a) and 5(b) show the changes in energy plotted as a function of the $x$ and $\theta$ coordinates, while Figs. 5(c) and 5(d) show the same variations plotted as a function of time $(t)$, normalized to the fundamental time period $(T)$. Figures 5(c) and 5(d) also show the total work done. These changes take place as the particle traverses the path shown by the curve marked $\mu / \mu_{X}=0.5$ in Fig. 4(d). Evidently, the optical force accelerates the particle over some regions of the cycle and acts as a brake, reducing kinetic energy, in other regions. Overall, the optical force increases the motional energy of the particle by an amount equal to that dissipated by viscous drag. The two processes can be seen to balance identically.
Figure 6 shows energy changes over limit cycles for various values of $\mu / \mu_{X}$ projected onto the $x$ and $\theta$ axes. The figure reveals the underlying symmetry of the energy changes. Since translational work depends on $\boldsymbol{v}$, through $v^{2}$, and not $\boldsymbol{\Omega}$, this quantity is an approximately single-valued function of $x$, i.e., for each value of $x$, there are two velocities, one positive and one negative, of approximately equal magnitude [Fig. 6(a)]. By comparison, it is a double-valued figure eight when plotted against $\theta$ since each value of $\theta$ corresponds to two different values of $v$, as the particle traverses the cycle [Fig. 6(b)]. The converse follows for rotational work Figs. 6(c) and 6(d).

In contrast to the case of the circular orbits, formed for isotropic spheres in circularly polarized beams [16], the limit cycles observed for birefringent spheres in linearly polarized beams are highly uneven with rates of dissipation, acceleration, and deceleration that vary substantially over the cycle. This observation has a number of implications which will be discussed in the last section.

Finally, we note that the use of an interpolated force field [rather than the approximations of Eqs. (2a) and (2b)] reproduces the fundamental frequencies [Fig. 4(b)] to within $1 \%$ and the Fourier amplitudes [Fig. 4(a)] to within $4 \%$.

\section{STOCHASTIC MOTION}

In the previous sections, we have shown that the stable fixed point of the deterministic system, which corresponds to the trapping position, destabilizes as the viscosity passes below a critical value $\mu_{X}$ and undergoes a Hopf bifurcation, leaving a limit cycle whose amplitude increases with decreasing viscosity. In the following section, we include thermal fluctuations and investigate the changes in the probability 

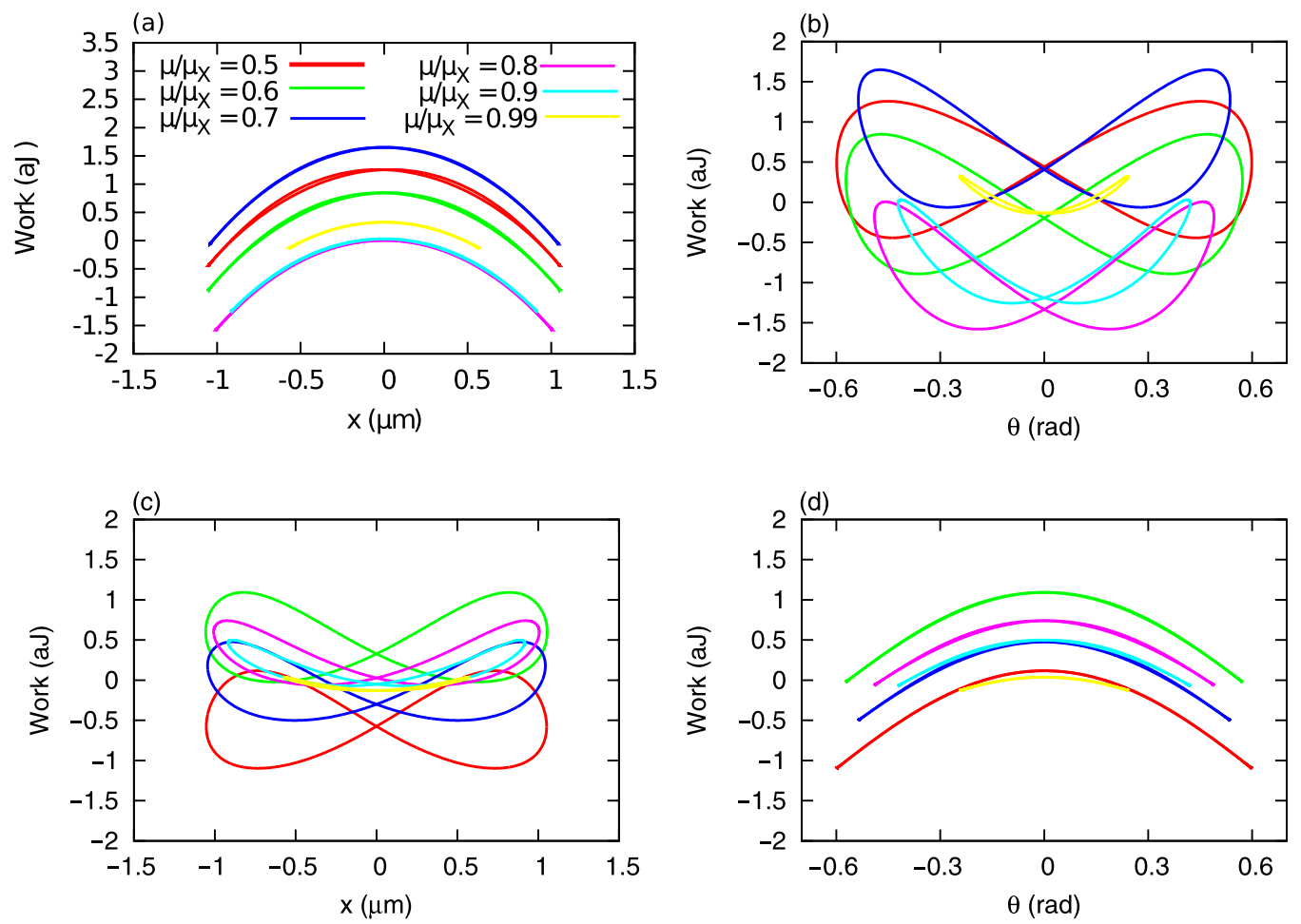

FIG. 6. Work done for (a), (b) translational and (c), (d) rotational projected onto the $x$ and $\theta$ axes (left and right columns, respectively). Limit cycles with varying values of $\mu / \mu_{X}<1$ are shown.

distribution function of the particle that accompany the approach to instability and the subsequent bifurcation of the underlying deterministic system. We start by considering the linearized force field, appropriate before the bifurcation, when $\mu>\mu_{X}$, and then progress to the general case.

\section{A. Linearized forces}

Returning to the linearized equations, given by Eq. (3), and including the thermal fluctuations, we have

$$
\begin{aligned}
-\mathbf{k} \mathbf{x}-\mathbf{\Xi} \dot{\mathbf{x}}+\mathbf{f}^{L}(t) & =\mathbf{M} \ddot{\mathbf{x}} \Longleftrightarrow\left(\mathbf{k}+i \omega \mathbf{\Xi}-\mathbf{M} \omega^{2}\right) \\
\mathbf{X} & \equiv \mathbf{A X}=\mathbf{F}^{L}(\omega) .
\end{aligned}
$$

Here, $\mathbf{F}^{L}(\omega)$ is the Fourier transform of the time-domain Langevin force, with zero mean and covariance proportional to the friction, i.e.,

$$
\begin{aligned}
\left\langle\mathbf{F}^{L}(\omega)\right\rangle & =0, \\
\left\langle\mathbf{F}^{L}(\omega) \otimes \mathbf{F}^{L}\left(\omega^{\prime}\right)\right\rangle & =2 k_{B} T \boldsymbol{\Xi} \delta\left(\omega-\omega^{\prime}\right) .
\end{aligned}
$$

The linearity of Eq. (9) ensures that the resulting steady state distribution function is normal [24],

$$
P(\overline{\mathbf{x}})=\frac{\exp \left[-\frac{1}{2}\left(\overline{\mathbf{x}}^{T} \mathbf{M} \overline{\mathbf{x}}\right)\right]}{4 \pi^{2} \sqrt{|\mathbf{C}|}},
$$

where $\overline{\mathbf{x}}=(x, \theta, \boldsymbol{v}, \boldsymbol{\Omega})$ are the complete phase-space coordinates, with $v=\dot{x}$ and $\boldsymbol{\Omega}=\dot{\theta}, \mathbf{C}$ is the covariance matrix, and its inverse $\mathbf{M}=\mathbf{C}^{-1}$. As described previously [17], $\mathbf{C}$ can be evaluated directly from Eqs. (9) and (10). The process can be summarized as follows. First, we write the right-hand side of Eq. (9) as $\mathbf{X}=\mathbf{A}^{-1} \mathbf{F}^{L}(\omega)$. Using Eq. (10), we evaluate the power spectral density (PSD), $\left\langle\mathbf{X}(\omega) \otimes \mathbf{X}^{*}(\omega)\right\rangle$. The
Wiener-Khinchin theorem then gives the covariance matrix C, as the Fourier transform of the PSD. This last step can be conveniently and accurately performed with residue calculus (see [16,17]). Previously, this procedure was used to find the covariance of the spatial coordinates (here, $x$ and $\theta$ ). However, it can be trivially extended to include velocities by observing that $\boldsymbol{v}=\dot{x}=i \omega x$ and $\boldsymbol{\Omega}=\dot{\theta}=i \omega \theta$. This gives the covariance of the velocities (e.g., $\left\langle\boldsymbol{v}(t) \boldsymbol{v}\left(t^{\prime}\right)\right\rangle$ ) as well as all cross terms (such as $\left\langle x(t) v\left(t^{\prime}\right)\right\rangle$ ). Elements of the covariance matrix $\mathbf{C}$ are obtained by setting $t=t^{\prime}$, e.g., $C_{x x}=\left\langle x^{2}\right\rangle$, and so on. $\mathbf{M}$ is obtained by direct inversion of $\mathbf{C}$. Having found $\mathbf{C}$ and $\mathbf{M}, P(\overline{\mathbf{x}})$ can be evaluated directly. Distributions over subsets of the coordinates can be found through integration, e.g., $P(x, \theta)=\iint P(x, \theta, \boldsymbol{v}, \boldsymbol{\Omega}) d \boldsymbol{v} d \boldsymbol{\Omega}$.

One noteworthy result of this analysis is that the variance in the coordinates diverges as $\mu \rightarrow \mu_{X}$. In particular,

$$
\left\langle x^{2}\right\rangle \propto \frac{1}{\omega_{X}^{2}\left(\mu-\mu_{X}\right)^{2}},
$$

where $\omega_{X}$ is given by Eq. (5) and $\left\langle\theta^{2}\right\rangle$ behaves similarly. We note that Eq. (12) is an artifact of the linear approximation. It provides a good approximation within the linear range of the trap, prior to the bifurcation, but fails thereafter. Having obtained the complete distribution function, given by Eq. (11), associated quantities can be found by direct integration. For instance, the integrated spatial probability currents [24],

$$
\begin{aligned}
& \left\langle S_{x}\right\rangle=\iint \boldsymbol{v} P(x, \theta, \boldsymbol{v}, \boldsymbol{\Omega}) d \boldsymbol{v} d \boldsymbol{\Omega}, \\
& \left\langle S_{\theta}\right\rangle=\iint \boldsymbol{\Omega} P(x, \theta, \boldsymbol{v}, \boldsymbol{\Omega}) d \boldsymbol{v} d \mathbf{\Omega},
\end{aligned}
$$




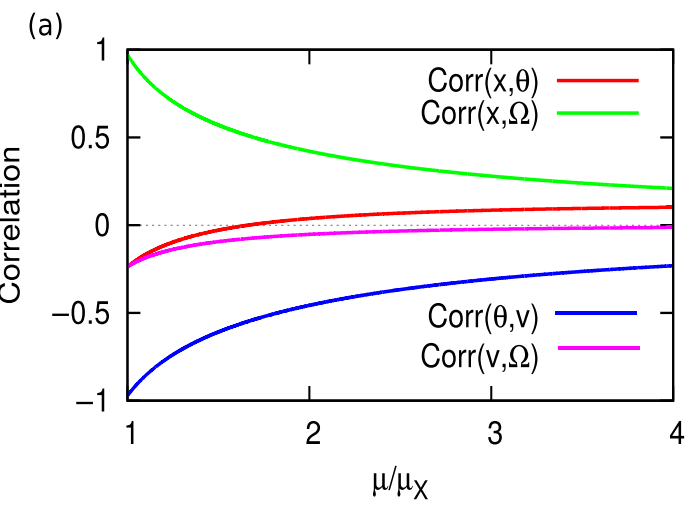

(b)

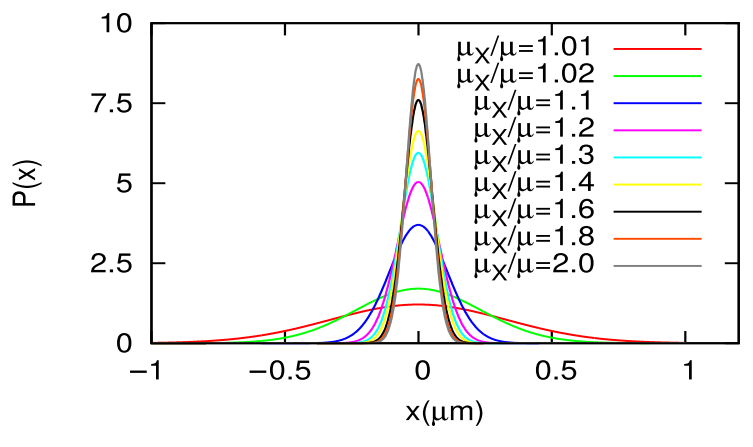

FIG. 7. (a) Nonzero cross correlations $\operatorname{Corr}(a, b)=\langle a b\rangle / \sqrt{\left\langle a^{2}\right\rangle\left\langle b^{2}\right\rangle}$ for variables $x, \theta, v$, $\boldsymbol{\Omega}$. As $\mu \rightarrow \mu_{X}$, Corr $(x, \boldsymbol{\Omega})$ approaches 1 and $\operatorname{Corr}(\theta, v)$ approaches -1 , as the bias towards deterministic cycling becomes stronger. (b) Analytical probability distribution $P(x)=$ $\iiint P(x, \theta, v, \boldsymbol{\Omega}) d \theta d \boldsymbol{v} d \boldsymbol{\Omega}$ for varying values of $\mu_{X} / \mu$ in the range $\mu / m u_{X}=2.0$ (highest peak) to $\mu / \mu_{X}=1.01$ (broadest peak).

are given by the following simple form:

$$
\left\langle S_{x / \theta}\right\rangle=-P(x, \theta)\left(F_{x / \theta} x+G_{x / \theta} \theta\right),
$$

where $P(x, \theta)$ is the spatial probability density, $P(x, \theta)=$ $\iint P(x, \theta, \boldsymbol{v}, \boldsymbol{\Omega}) d \boldsymbol{v} d \boldsymbol{\Omega}$, which, once more, is normal. The scalar quantities $F_{x / \theta}, G_{x / \theta}$ and the covariance matrix of $P(x, \theta)$ are completely determined by $\mathbf{C}$ (and its inverse, $\mathbf{M}$ ), i.e., these quantities are independent of the coordinates $x$ and $\theta$. Explicit expressions are provided in the Appendix. The complete set of currents, including $S_{v}$ and $S_{\boldsymbol{\Omega}}$, enters into the continuity equation for the probability density, i.e., the Klein-Kramers equation [24]. They describe flows of probability within the distribution. An immediate consequence of Eq. (14) is that the mean velocities, $\langle v\rangle=\left\langle S_{x}\right\rangle / P(x, \theta)$ and $\langle\boldsymbol{\Omega}\rangle=\left\langle S_{\theta}\right\rangle / P(x, \theta)$, are linear in the coordinates, $(x, \theta)$, e.g., $\langle v\rangle(a x, a \theta)=a\langle v\rangle(x, \theta)$, for scalar constant $a$. In other words, $\langle v\rangle$ and $\langle\boldsymbol{\Omega}\rangle$ increase linearly along position vectors in $x / \theta$ space, although the probability density $P(x, \theta)$, which is normal, decreases rapidly.

Numerical results for the default parameters are shown in Figs. 7 and 8. Figure 7(a) shows nonzero elements of the correlation matrix (derived from the covariance matrix by normalizing, e.g., $\left.\operatorname{Corr}(x, \theta)=\langle x \theta\rangle / \sqrt{C_{x x} C_{\theta \theta}}\right)$. Some elements of the covariance matrix and, therefore, the correlation matrix vanish identically. For example, $\langle x v\rangle \equiv 0$ since, for any fixed value of $x$, the velocity is as likely to be positive as negative. If this were not true, the distribution would not be stationary. Similarly, $\langle\theta \boldsymbol{\Omega}\rangle \equiv 0$. Other elements, such as $\langle x \boldsymbol{\Omega}\rangle$ and $\langle\theta v\rangle$, take finite values. Figure $7(\mathrm{~b})$ shows the integrated probability distribution function, $P(x)=\iiint P(x, \theta, v, \boldsymbol{\Omega}) d \theta d \boldsymbol{v} d \boldsymbol{\Omega}$, evaluated from Eq. (11) by formal integration (see the Appendix). As can be seen, $P(x)$ spreads out as the viscosity is reduced towards the critical value $\mu_{X}$. We note that for $\mu=\mu_{X}$, elements of the covariance matrix become infinite, as the imaginary part of one of the characteristic frequencies reaches zero $[16,17]$ and the corresponding distribution loses validity. These results should be contrasted with the case for motion in a potential. In this case, the Boltzmann distribution is attained. The variances of $x$ and $\theta$ are determined by the potential, according to the equipartition theorem, as are the variances in the translational and rotational velocities, which are $\left\langle v^{2}\right\rangle=k_{B} T / m$ and $\left\langle\boldsymbol{\Omega}^{2}\right\rangle=k_{B} T / I$, respectively. For motion in a potential, then, the covariance matrix and the corresponding distribution function are completely independent of viscosity. In addition, correlations between positions and velocities are necessarily zero for the Boltzmann distribution, in contrast to the nonconservative system studied here. This latter point underpins Fig. 8, which shows the integrated spatial probability currents [see Eqs. (13) and (14) and the Appendix) as a vector field, $\left(\left\langle S_{x}\right\rangle(x, \theta),\left\langle S_{\theta}\right\rangle(x, \theta)\right)$, superposed onto the integrated probability distribution $P(x, \theta)=$ $\iint P(x, \theta, \boldsymbol{v}, \boldsymbol{\Omega}) d \boldsymbol{v} d \boldsymbol{\Omega}$. These quantities relate to mean velocities via $\langle v\rangle=\left\langle S_{x}\right\rangle / P(x, \theta)$ and similarly for $\langle\boldsymbol{\Omega}\rangle$. Again, for a conservative system, the probability currents vanish identically [24]. Here, they swirl around the trapping point (Fig. 8). As the viscosity is reduced towards the critical value, the currents reduce in magnitude but spread over greater regions of space. Interestingly, for high viscosities, e.g., $\mu / \mu_{X} \approx 2$, the currents persist, even though the distribution function approaches the shape of the Boltzmann distribution (Fig. 9); as the viscosity approaches the overdamped regime, the probability currents have less and less influence over the particle momentum and, consequently, the shape of the distribution function.

\section{B. Stochastic bifurcation}

Finally, we consider the general system including nonlinearities in the force field and thermal fluctuations. In particular, we numerically integrate the Langevin equation, given by Eq. (1), with the force field, given by Eq. (2), using the scheme described previously $[17,30]$. Figure 9 shows graphs of the probability distribution in one dimension, the remaining dimensions having been integrated out. Figures 9(a) and $9(\mathrm{c})$ relate to viscosities above the critical value (i.e., $\mu>\mu_{X}$ ), and Figs. 9(b) and 9(d) are after the bifurcation (i.e., $\left.\mu<\mu_{X}\right)$. The top pair is plotted against the $x$ coordinate and the lower pair against $v$.

Figures 9(a) and 9(c) $\left(\mu>\mu_{X}\right)$ include analytical curves relating to the Boltzmann distribution that would be obtained for a conservative system described by the linear terms in the force field (2), with coupling coefficients $k_{\theta}^{(x)}$ and $k_{x}^{(\theta)}$ set to zero. For each numerical curve (continuous lines), we have included an analytical approximation (dashed lines) obtained using the approach described above, for the linearized nonconservative system. The probability distributions increas- 

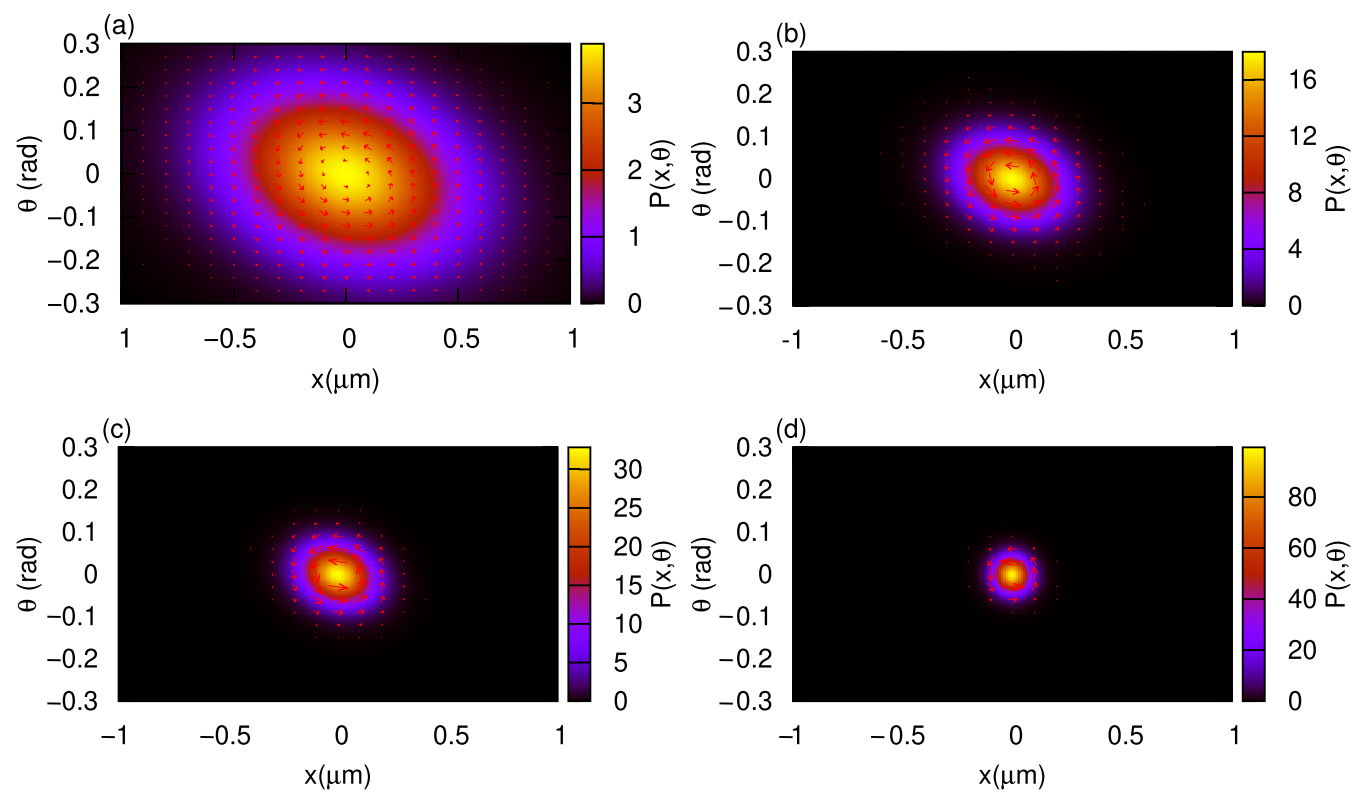

FIG. 8. Integrated probability distributions $P(x, \theta)=\iint P(x, \theta, \boldsymbol{v}, \boldsymbol{\Omega}) d \boldsymbol{v} d \boldsymbol{\Omega}$ and probability currents $\langle\mathbf{S}\rangle$ for $\mu / \mu_{X}=($ a) 1.01 , (b) 1.05 , (c) 1.1, (d) 1.5. $P(x, \theta)$ is shown as a heat map and the vector field $\langle\mathbf{S}\rangle$ is represented by the red arrows. The currents swirl counterclockwise about the origin.

ingly resemble the Boltzmann distribution as $\mu$ increases [Figs. 9(a) and 9(c)]. As discussed above, the similarity in the shapes of the distribution functions should not be confused with a qualitative similarity between the distributions themselves: The covariance matrix of the nonconservative system contains nonzero elements, such as $\langle x \boldsymbol{\Omega}\rangle$ and $\langle\theta v\rangle$, which do not appear in the Boltzmann distribution in which the probability currents, present in the nonconservative case, also vanish. For higher values of $\mu$, the analytical and numerical distribution functions [Figs. 9(a) and 9(c)] agree closely and the lines for the numerical and analytical results are superposed. As before, reducing $\mu$ causes the distributions to
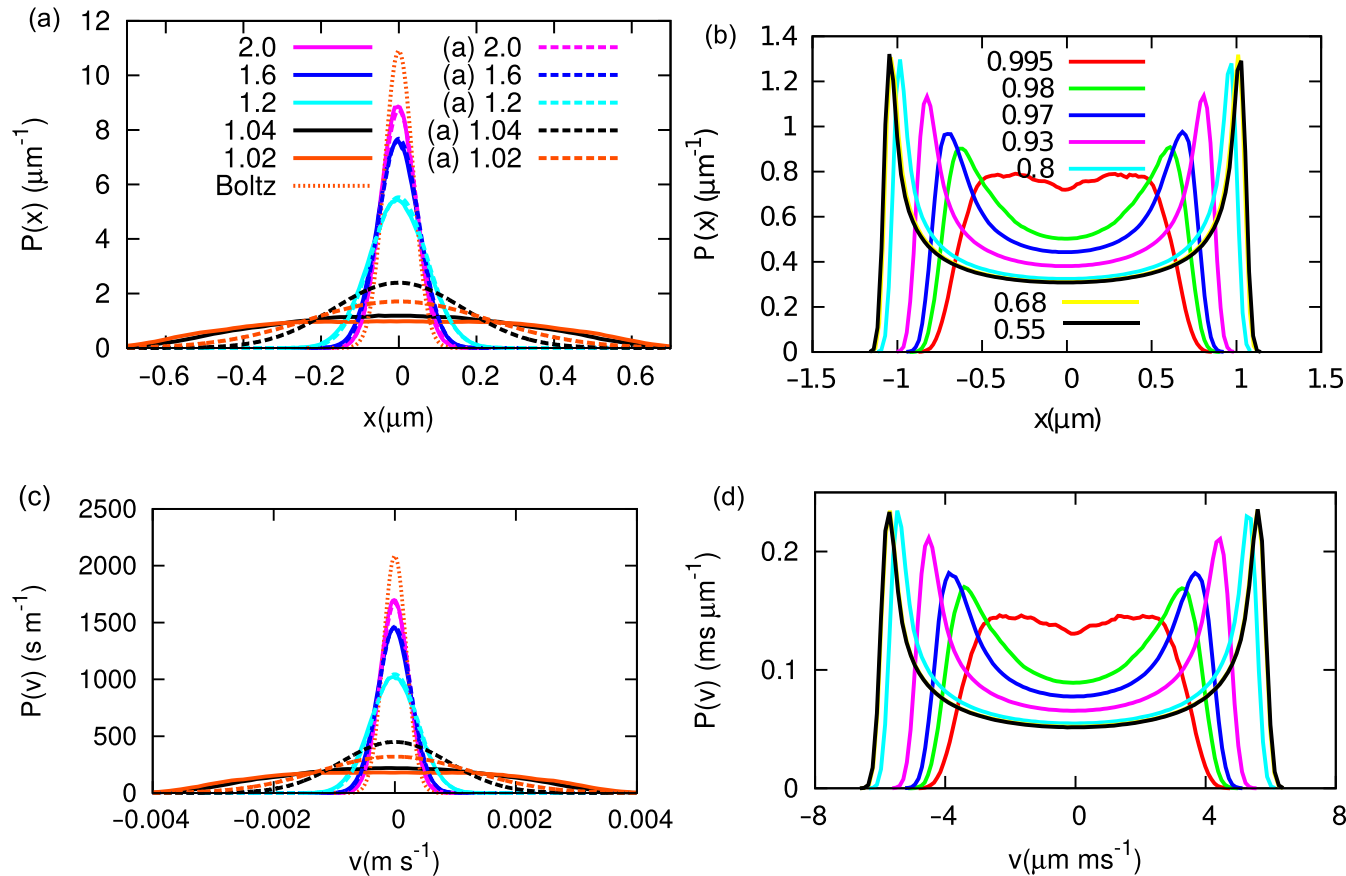

FIG. 9. Simulated 1D distribution functions for viscosities (a), (c) above and (b), (d) below the critical value. (a), (b) $P(x)$; (c), (d) $P(v)$. Different values of $\mu / \mu_{X}$ are plotted in different colors. Continuous lines correspond to numerical simulations and dotted lines are analytical approximations for an equivalent linearized system. In general, decreasing $\mu / \mu_{X}$ corresponds to broadening distributions. For viscosities substantially greater than $\mu_{X}$, the distributions are approximately Boltzmann. Results for $\theta$ and $\Omega$ are similar to those of $x$ and $v$. 

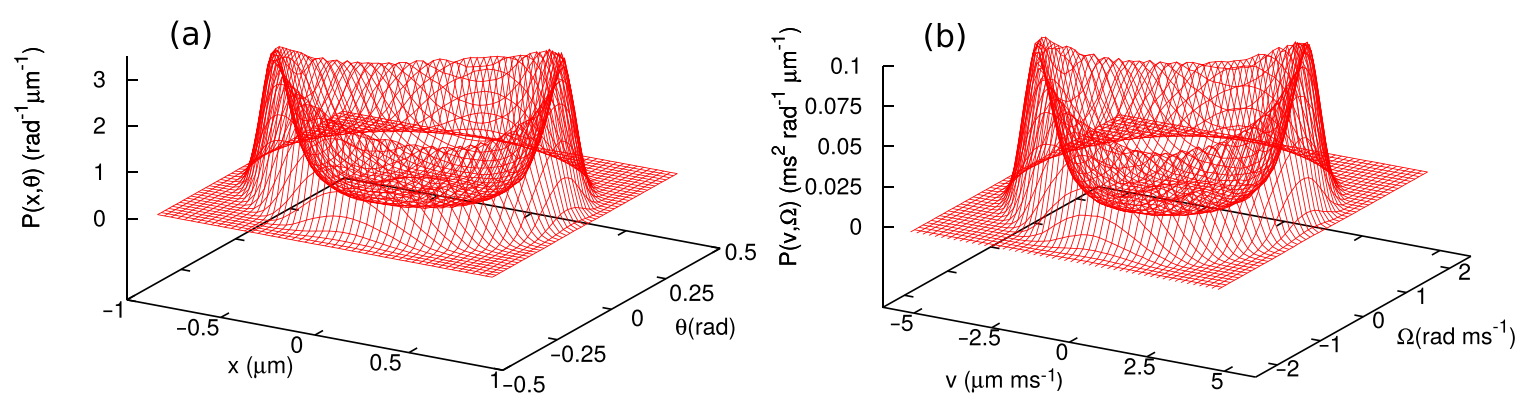

FIG. 10. Two-dimensional probability distributions for $\mu / \mu_{X}=0.985$, (a) $P(x, \theta)$ and (b) $P(x, \boldsymbol{\Omega})$.

spread out along both $x$ and $v$. As they do so, the numerical distribution functions depart from the analytical approximations, which become comparatively broad. This discrepancy is caused by the fact that for viscosities approaching the critical value $\mu_{X}$, the particle motion can exceed the linear range of the trap [see Eq. (12)], and nonlinearities in the force field are increasingly significant. In effect, the trajectories tend increasingly toward stable limit cycles, rather than outward spirals, and this serves to confine the particle more strongly than would be expected from the linearized equations of motion.

Figures 9(b) and 9(d) show the corresponding distributions for $\mu<\mu_{X}$. Immediately after the bifurcation of the deterministic system, the distribution functions are quite flat. As $\mu$ is further decreased, double-peaked distributions emerge, and the peaks becoming progressively sharp with decreasing $\mu$. The two-dimensional distributions $P(x, \theta)$ and $P(v, \boldsymbol{\Omega})$ reveal more about the process (Fig. 10). As $\mu$ is decreased, more and more energy is associated with the underlying limit cycles, exceeding the thermal energy $k_{B} T / 2$. The motion becomes progressively more deterministic and the thermal fluctuations, taking the particle away from the limit cycle, become relatively less significant. The system is a noisy, self-sustained oscillator, fluctuating around an uneven limit cycle in phase space.

Finally, we note that the bifurcation in the deterministic system is associated with an abrupt change in the kurtosis of the probability distribution function, from a value 3 , when $\mu>\mu_{X}$ (corresponding to the condition for normal distributions) to a value of 1.5 when $\mu<\mu_{X}$. Figure 11 explores this observation for a range of parameters, beyond the default set used previously. In Fig. 11(a), we plot the variance in the position $\left\langle x^{2}\right\rangle$ for birefringent particles with differing radii. Variations in $\left\langle x^{2}\right\rangle$ are loosely concentrated around $\mu=\mu_{X}$, but the changes are smooth and continuous, and the behavior for $\mu<\mu_{X}$ varies with particle size. Figure 11(c) shows the corresponding variations in kurtosis, showing the abrupt transition between two constant values, a zoomed-in version of which is given in Fig. 11(d). We observe that the change in kurtosis is relatively smooth at this scale, although the range of absolute viscosities is quite narrow. The numerical simulations often show unusual behavior close to $\mu=\mu_{X}$, an extreme example of which appears in Fig. 11(d), for sphere radius $a=2.3 \mu \mathrm{m}$, where the kurtosis is seen to spike upward, acquiring a value of $\approx 4.25$. This effect appears when $\mu$ is slightly greater than $\mu_{X}$. For this narrow range of viscosities, the trapping point is on the verge of destabilizing and the linear approximation indicates a rapidly diverging variance, given by Eq. (12), exposing the particle to nonlinearities in the force field even though the limit cycles are not yet stable themselves. Lastly, Fig. 11(b) shows the kurtosis for the other coordinates, $\theta, v$, and $\boldsymbol{\Omega}$, confirming that a similar transition takes place for all coordinates.

\section{DISCUSSION}

In this article, we have provided a detailed account of the motion of a birefringent microsphere in a linearly polarized Gaussian optical trap, in vacuum.

When thermal fluctuations are ignored, the underlying deterministic motion undergoes a Hopf bifurcation when the viscosity $\mu$ is reduced below a critical value $\mu_{X}$. For viscosities above $\mu_{X}$, the system has a stable fixed point. However, the forces and torques acting on the particle are linearly nonconservative, due to nonsymmetric rotation-translation coupling. As the viscosity is reduced below the critical value, the trapping point is destabilized. Any perturbation away from it results in the particle trajectory spiraling into a stable limit cycle consisting of a closed loop in the position and angle coordinates $x$ and $\theta$. The limit cycles are highly uneven, with the velocity and angular velocity varying significantly over the cycle. Further reductions in viscosity have a weak influence on the fundamental frequency of the cycle, but increase its amplitude.

When thermal effects are included in this description, the bifurcation corresponds to a change from one regime, consisting of fluctuations around a stable fixed point, to another, which consists of fluctuations around a stable limit cycle. In the first regime $\left(\mu>\mu_{X}\right)$, the particle is confined by linear terms in the force field and the probability distribution is normal, to good approximation. It differs from the Boltzmann distribution in several respects. For example, the covariance matrix contains cross terms (such as $\langle x \boldsymbol{\Omega}\rangle$ and $\langle\theta v\rangle$ ), which would otherwise be zero. In addition, the width of the distribution increases sharply as $\mu \rightarrow \mu_{X}$. This is accompanied by increasing coherence and a sharpening peak in the power spectral density [17], in a manner resembling coherence resonance $[35,36]$. Associated with this, persistent probability currents swirl about the stable fixed point. For conservative systems, these currents would be completely absent. After bifurcation $\left(\mu<\mu_{X}\right)$, the probability distribution function flattens and broadens, forming double peaks for the one-dimensional (1D) distributions, $P(x)$ and $P(\theta)$. In two dimensions, $P(x, \theta)$ is increasingly concentrated in an oblique elliptical ring, reflecting the limit cycle of the deterministic 

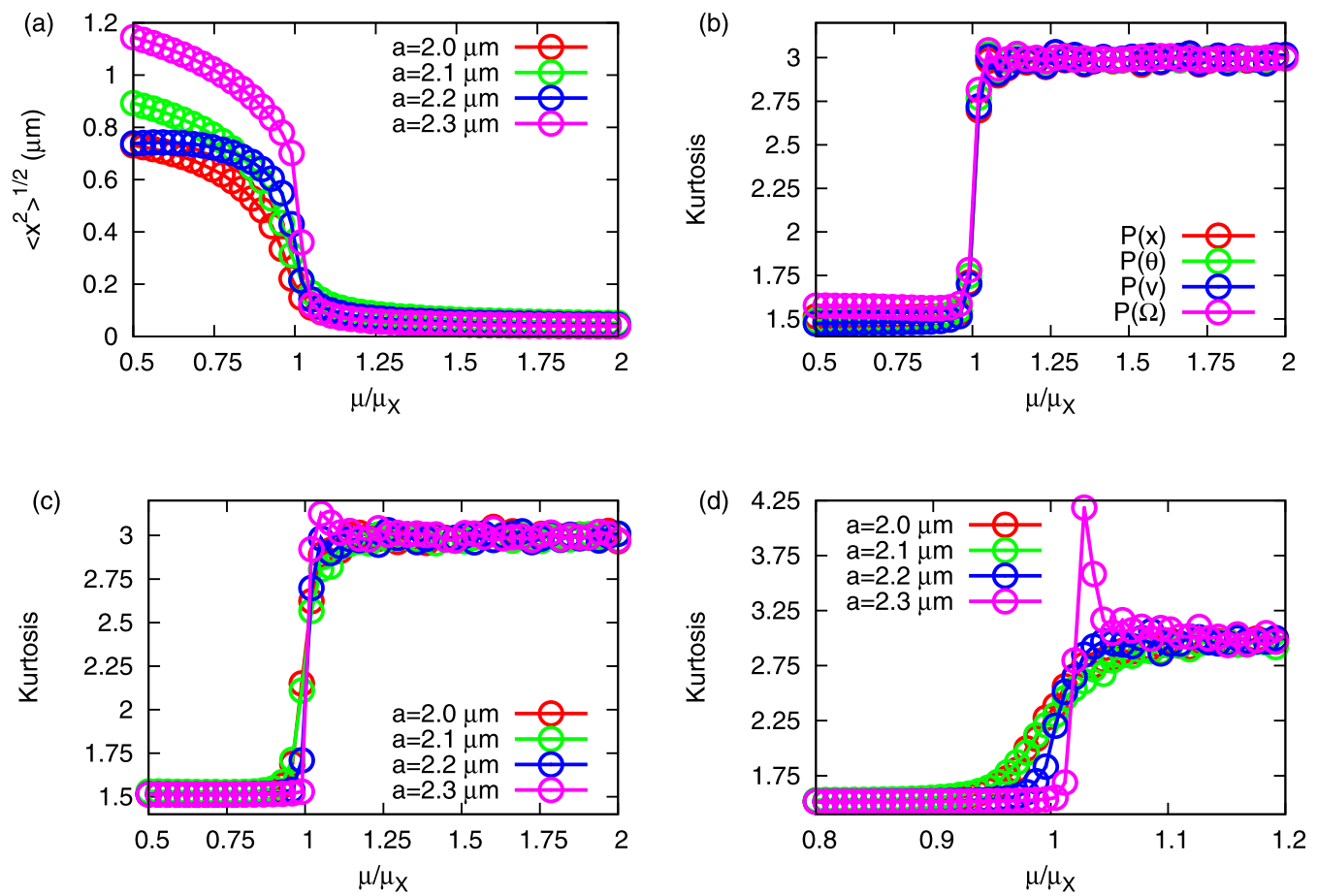

FIG. 11. (a) Variation in $\sqrt{\left\langle x^{2}\right\rangle}$ with $\mu / \mu_{X}$ for spheres of various radii. Kurtosis as a function of $\mu / \mu_{X}$ for (b) the integrated distribution functions, $P(x), P(v), P(\theta)$, and $P(\boldsymbol{\Omega})$, using the default parameters, and (c), (d) for $P(x)$ with spheres of various radii. (d) Zoomed-in version of (c).

motion. The bifurcation is associated with a sharp change in the kurtosis of the distribution function from a value of 3 (for the normal distribution, when $\mu>\mu_{X}$ ) to 1.5 thereafter.

Although the preceding account has been quite general, we have neglected to discuss what happens as the viscosity is decreased much below $\mu_{X}$. Two qualitative comments can be made. First, the limit cycles formed for $\mu<\mu_{X}$ must destabilize at some point. Appropriate conditions can be found through analysis of the Poincaré map [37]. For the system considered here, the limit cycles remain stable for the range of parameters considered above. Second, the influence of vertical (z) optical forces has been neglected. These forces are even in the $x$ coordinate, i.e., $\sim x^{2}$, and act on the particle increasingly as the amplitude of the $x$ oscillation increases. As revealed in detailed simulations (e.g., [17]), this effect is relatively weak for the range of parameters considered here. However, as $x$ takes increasingly larger values, the vertical force, which will oscillate at about twice the fundamental frequency of the limit cycle, will start to produce nonperiodic motion. These theoretical considerations might be contrasted with what actually happens in the laboratory. As shown in [17], double-peaked distributions are readily observed, indicating the formation of a limit cycle. However, decreasing the viscosity much below this point results in the rapid loss of the particle from the trap and these more complex motions, associated with the collapse of the limit cycle or the interference of the $z$ force, are hard to investigate in the laboratory.

This system has a number of interesting and potentially useful properties. First, it provides an opportunity for directly observing fluctuations in a bifurcating system with a nonconservative instability. Such systems are representative of a broader physical class including, for instance, firing of neurons [25,26], laser dynamics [27,28], and electrochemical reactions [38]. Although we have covered the salient properties of this system, there remains much to understand concerning the detailed stochastic dynamics, entropy production [39-41], and so on. Second, the unevenness of the limit cycles lends itself to various kinds of synchronization $[42,43]$ (which, in this case, may be mediated by optical interactions, e.g., Ref. [44]) and resonance effects, including coherence resonance [36,38] (which occurs during the approach to a bifurcation) and autonomous stochastic resonance [45,46] (in which motion about a limit cycle synchronizes with weak periodic modulations in the external force), both of which are exhibited by single neurons and neuronal networks (e.g., $[47,48])$. The latter phenomenon, autonomous stochastic resonance, may explain the response of oscillating birefringent spheres to parametric forcing that has been observed previously [17].

The optomechanical limit cycle oscillators described above may be designed or refined with combinations of beam shaping technology [49] and tailored optical interactions [44,50]. More detailed investigation of their stochastic dynamics, especially with regard to coherence resonance, autonomous stochastic resonance, and synchronization, could lead to novel applications in sensing and metrology, while advanced cooling protocols (e.g., [51,52]) could allow these effects to be pushed towards the quantum regime [53-55].

\section{ACKNOWLEDGMENTS}

We acknowledge the support from the Engineering and Physical Sciences Research Council (Grant No. EP/P030017/1), the European Regional Development Fund 
(Grant No. CZ.02.1.01/0.0/0.0/15_003/0000476), the Czech Science Foundation (Grant No. GA19-17765S), and the Czech Academy of Sciences (Praemium Academiae, Grant No. RVO:68081731).

\section{APPENDIX}

\section{Force field}

In Table I we provide the numerical values for the approximate forces and torques, given by Eq. (2). The column headed $(x)$ lists coefficients for the force, given by Eq. (2a), and the column headed ${ }^{(\theta)}$ lists coefficients for the torque, given by Eq. (2b). These coefficients give the force [Eq. (2a)] in pN
TABLE I. Numerical values for the parameters in the approximate force field, Eqs. (2a) and (2b).

\begin{tabular}{lcr}
\hline \hline & $(x)$ & \multicolumn{1}{c}{$(\theta)$} \\
\hline$k_{x}$ & 3.095 & -1.2125 \\
$k_{\theta}$ & 1.082 & 6.8904 \\
$\chi_{0}$ & 0.8165 & -0.2172 \\
$\chi_{1}$ & 0.1939 & 0.0423 \\
$\chi_{2}$ & 0.9552 & 1.8317 \\
$\chi_{3}$ & -1.2878 & -3.9383 \\
\hline \hline
\end{tabular}

and the torque [Eq. (2b)] in $\mathrm{pN} \mu \mathrm{m}$, for displacements $x$ in $\mu \mathrm{m}$ and rotations in radians.

\section{Probability distributions and currents}

The spatial probability distribution and integrated spatial probability currents are given in terms of the covariance matrix $\mathbf{C}$ and its inverse $\mathbf{M}$ as follows:

$$
\begin{gathered}
P(x, \theta)=\iint P(x, \theta, \boldsymbol{v}, \boldsymbol{\Omega}) d \boldsymbol{v} d \boldsymbol{\Omega}=\frac{E_{1}(x, \theta) E_{2}(x, \theta) E_{3}(x, \theta)}{2 \pi \sqrt{|\mathbf{C}|} \sqrt{M_{v v} M_{\boldsymbol{\Omega} \boldsymbol{\Omega}}-M_{v \boldsymbol{\Omega}}^{2}}}, \\
\left\langle S_{x}\right\rangle=-P(x, \theta)\left[F_{x} x+G_{x} y\right], \\
\left\langle S_{\theta}\right\rangle=-P(x, \theta)\left[F_{y} x+G_{y} y\right],
\end{gathered}
$$

where

$$
\begin{gathered}
E_{1}(x, \theta)=\exp \left[-\frac{1}{2}\left(M_{x x} x^{2}+M_{\theta \theta} \theta^{2}+2 M_{x \theta} x \theta\right)\right], \\
E_{2}(x, \theta)=\exp \left[\left(M_{x v}^{2} x^{2}+M_{\theta v}^{2} \theta^{2}+2 M_{x v} M_{\theta v} x \theta\right) /\left(2 M_{v v}\right)\right], \\
E_{3}(x, \theta)=\exp \left\{\frac{\left[\left(M_{x \boldsymbol{\Omega}} M_{v v}-M_{x v} M_{v \boldsymbol{\Omega}}\right) x+\left(M_{\theta \boldsymbol{\Omega}} M_{v v}-M_{\theta v} M_{v \boldsymbol{\Omega}}\right) y\right]^{2}}{2 M_{v v}\left(M_{v v} M_{\boldsymbol{\Omega} \boldsymbol{\Omega}}-M_{v \boldsymbol{\Omega}}^{2}\right)}\right\},
\end{gathered}
$$

and

$$
\begin{gathered}
F_{x}=\left(M_{x v} M_{\boldsymbol{\Omega} \Omega}-M_{v \boldsymbol{\Omega}} M_{x \boldsymbol{\Omega}}\right) / D, \\
G_{x}=\left(M_{\theta v} M_{\boldsymbol{\Omega} \boldsymbol{\Omega}}-M_{v \boldsymbol{\Omega}} M_{\theta \boldsymbol{\Omega}}\right) / D, \\
F_{\theta}=\left(M_{x \boldsymbol{\Omega}} M_{v v}-M_{v \boldsymbol{\Omega}} M_{x v}\right) / D, \\
G_{\theta}=\left(M_{\theta \boldsymbol{\Omega}} M_{v v}-M_{v \boldsymbol{\Omega}} M_{\theta v}\right) / D,
\end{gathered}
$$

with

$$
D=M_{v v} M_{\Omega \Omega}-M_{v \Omega}^{2}
$$

[1] K. Dholakia and T. Cizmar, Nat. Photon. 5, 335 (2011).

[2] P. Zemánek, G. Volpe, A. Jonáš, and O. Brzobohatý, Adv. Opt. Photon. 11, 577 (2019).

[3] K. Neuman and S. Block, Rev. Sci. Instrum. 75, 2787 (2004).

[4] R. W. Bowman and M. J. Padgett, Rep. Prog. Phys. 76, 026401 (2013).

[5] K. Berg-Sorensen and H. Flyvbjerg, Rev. Sci. Instrum. 75, 594 (2004).

[6] A. Rohrbach and E. Stelzer, Appl. Opt. 41, 2494 (2002).
[7] S. H. Simpson and S. Hanna, Phys. Rev. E 82, 031141 (2010).

[8] A. Irrera, A. Magazzú, P. Artoni, S. H. Simpson, S. Hanna, P. H. Jones, F. Priolo, P. G. Gucciardi, and O. M. Maragó, Nano Lett. 16, 4181 (2016).

[9] I. Ortega-Piwonka, C. N. Angstmann, B. I. Henry, and P. J. Reece, Chaos 28, 043101 (2018).

[10] W. J. Toe, I. Ortega-Piwonka, C. N. Angstmann, Q. Gao, H. H. Tan, C. Jagadish, B. I. Henry, and P. J. Reece, Phys. Rev. E 93, 022137 (2016). 
[11] S. H. Simpson, P. Zemánek, O. M. Maragò, P. H. Jones, and S. Hanna, Nano Lett. 17, 3485 (2017).

[12] P. Polimeno, M. Iatì, C. D. E. Boschi, S. Simpson, V. Svak, O. Brzobohatý, P. Zemánek, O. Maragò, and R. Saija, Eur. Phys. J. Plus 136, 1 (2021).

[13] P. Wu, R. Huang, C. Tischer, A. Jonas, and E.-L. Florin, Phys. Rev. Lett. 103, 108101 (2009).

[14] B. Suassuna, B. Melo, and T. Guerreiro, Phys. Rev. A 103, 013110 (2021).

[15] Y. Roichman, B. Sun, A. Stolarski, and D. G. Grier, Phys. Rev. Lett. 101, 128301 (2008).

[16] V. Svak, O. Brzobohatý, M. Šiler, P. Jákl, J. Kaňka, P. Zemánek, and S. Simpson, Nat. Commun. 9, 5453 (2018).

[17] Y. Arita, S. H. Simpson, P. Zemanek, and K. Dholakia, Sci. Adv. 6, eaaz9858 (2020).

[18] S. H. Simpson, S. Hanna, T. J. Peterson, and G. A. Swartzlander, Opt. Lett. 37, 4038 (2012).

[19] D. B. Phillips, M. J. Padgett, S. Hanna, Y. L. D. Ho, D. M. Carberry, M. J. Miles, and S. H. Simpson, Nat. Photon. 8, 400 (2014).

[20] S. Kuhn, B. A. Stickler, A. Kosloff, F. Patolsky, K. Hornberger, M. Arndt, and J. Millen, Nat. Commun. 8, 1 (2017).

[21] M. Rashid, M. Toroš, A. Setter, and H. Ulbricht, Phys. Rev. Lett. 121, 253601 (2018).

[22] T. M. Hoang, Y. Ma, J. Ahn, J. Bang, F. Robicheaux, Z. Q. Yin, and T. C. Li, Phys. Rev. Lett. 117, 123604 (2016).

[23] B. A. Stickler, S. Nimmrichter, L. Martinetz, S. Kuhn, M. Arndt, and K. Hornberger, Phys. Rev. A 94, 033818 (2016).

[24] H. Risken, The Fokker-Planck Equation: Methods of Solution and Applications (Springer-Verlag, Berlin, 1989), Chap. 3.

[25] A. Longtin, J. Stat. Phys. 70, 309 (1993).

[26] A. Longtin, Phys. Rev. E 55, 868 (1997).

[27] S. Wieczorek, Phys. Rev. E 79, 036209 (2009).

[28] O. V. Ushakov, H.-J. Wünsche, F. Henneberger, I. A. Khovanov, L. Schimansky-Geier, and M. Zaks, Phys. Rev. Lett. 95, 123903 (2005).

[29] S. Beresnev, V. Chernyak, and G. Fomyagin, J. Fluid Mech. 219, 405 (1990).

[30] N. Gronbech-Jensen and O. Farago, Mol. Phys. 111, 983 (2013).

[31] J. Roorda and S. Nemat-Nasser, AIAA J. 5, 1262 (1967).
[32] S. H. Simpson and S. Hanna, J. Opt. Soc. Am. A 26, 625 (2009).

[33] A. Doicu, T. Wreidt, and Y. A. Eremin, Light Scattering by Systems of Particles (Springer, New York, 2006).

[34] Mathematica, Ver. 12.0 (W. R. Inc., Champaign, IL, 2018).

[35] A. S. Pikovsky and J. Kurths, Phys. Rev. Lett. 78, 775 (1997).

[36] A. Neiman, P. I. Saparin, and L. Stone, Phys. Rev. E 56, 270 (1997).

[37] S. Wiggins, Introduction to Applied Nonlinear Dynamical Systems and Chaos (Springer, New York, 1990).

[38] I. Z. Kiss, J. L. Hudson, G. J. Escalera Santos, and P. Parmananda, Phys. Rev. E 67, 035201(R) (2003).

[39] T. J. Xiao, Z. Hou, and H. Xin, J. Chem. Phys. 129, 114506 (2008)

[40] S. Lahiri and A. Jayannavar, Eur. Phys. J. B 69, 87 (2009).

[41] S. J. Box, M. P. Allen, D. B. Phillips, and S. H. Simpson, Front. Phys. 8, 538 (2020).

[42] L. Callenbach, P. Hänggi, S. J. Linz, J. A. Freund, and L. Schimansky-Geier, Phys. Rev. E 65, 051110 (2002).

[43] M. Komarov, S. Gupta, and A. Pikovsky, Europhys. Lett. 106, 40003 (2014).

[44] V. Svak, J. Flajšmanová, L. Chvátal, M. Šiler, A. Jonáš, J. Ježek, S. H. Simpson, P. Zemánek, and O. Brzobohatý, Optica 8, 220 (2021).

[45] Hu Gang, T. Ditzinger, C.-Z. Ning, and H. Haken, Phys. Rev. Lett. 71, 807 (1993).

[46] W.-J. Rappel and S. H. Strogatz, Phys. Rev. E 50, 3249 (1994).

[47] B. Sancristóbal, B. Rebollo, P. Boada, M. V. Sanchez-Vives, and J. Garcia-Ojalvo, Nat. Phys. 12, 881 (2016).

[48] D. Guo and C. Li, Phys. Rev. E 79, 051921 (2009).

[49] F. M. Dickey, Laser Beam Shaping: Theory and Techniques (CRC Press, Boca Raton, FL, 2018).

[50] Y. Arita, E. M. Wright, and K. Dholakia, Optica 5, 910 (2018).

[51] U. Delić, M. Reisenbauer, K. Dare, D. Grass, V. Vuletić, N. Kiesel, and M. Aspelmeyer, Science 367, 892 (2020).

[52] M. Aspelmeyer, T. J. Kippenberg, and F. Marquardt, Rev. Mod. Phys. 86, 1391 (2014).

[53] L. Ben Arosh, M. C. Cross, and R. Lifshitz, Phys. Rev. Research 3, 013130 (2021)

[54] Y. Kato and H. Nakao, New J. Phys. 23, 043018 (2021).

[55] M. Koppenhöfer and A. Roulet, Phys. Rev. A 99, 043804 (2019). 